\title{
AVALIAÇÃO DO REPERTÓRIO COMPORTAMENTAL DE BEBÊS NOS QUATRO PRIMEIROS MESES DE VIDA: UMA PROPOSTA DE ANÁLISE
}

\author{
EVALUATION OF THE BEHAVIORAL REPERTOIRE OF BABIES IN THE FIRST \\ FOUR MONTHS OF LIFE: AN ANALYSIS PROPOSAL
}

Dora Carolina Silva Ribeiro Taques*

Olga Maria Piazentin Rolim Rodrigues**

\begin{abstract}
Taques DCSR, Rodrigues OMPR. Avaliação de repertório comportamental de bebês nos quatro primeiros meses de vida: uma proposta de análise. Rev Bras Crescimento Desenvolv Hum.2006; 16(2):77-87.
\end{abstract}

Resumo: O presente projeto avaliou o desenvolvimento de 40 bebês, utilizando o Inventário Portage Operacionalizado (IPO), durante os primeiros quatro meses de vida. Neste período foi avaliada a ocorrência, a cada mês, de 45 comportamentos, previstos no protocolo de Estimulação Infantil. Esta avaliação pretendeu identificar diferenças de freqüência destes comportamentos a cada mês. As avaliações mensais ocorreram em data próximo ao aniversário de mês de cada bebê, em agendamento prévio. Os bebês eram oriundos de uma maternidade que atende, prioritariamente, usuárias do SUS. Os critérios de análise foram: condições de estabilização (ocorrência para mais de $75 \%$ da amostra), normalização (de 25\% a $75 \%$ da amostra) e aparecimento (menos de $25 \%$ dos bebês) a cada mês. Os resultados obtidos apontaram para a existência de comportamentos típicos (estabilizados) e prováveis (normalizados) para cada mês. No primeiro mês, os bebês apresentaram $25 \%$ (estabilização) dos 45 comportamentos avaliados. Do restante deles, os bebês apresentaram $26 \%$ no segundo mês. No terceiro mês, dos comportamentos que não estavam ainda estabilizados os bebês apresentaram 36\% e, no quarto mês apresentaram $44 \%$ dos que restaram. Tais resultados permitem concluir que um número menor de comportamentos avaliados facilitaria sobremaneira 0 processo de avaliação de bebês e orientação de pais, resultando em economia de tempo e desgaste emocional dos cuidadores e aplicadores. Estudos conduzidos com populações maiores poderão validar estes resultados.

Palavras-chave: Inventário Portage Operacionalizado. Desenvolvimento de bebês. Avaliação de desenvolvimento.

\section{INTRODUÇÃO}

Os avanços da tecnologia têm favorecido a sobrevida de crianças nascidas em condições adversas. Dessa forma, vem ocorrendo um aumento no número de alterações de desenvolvimento, que devem ser diagnosticadas cada vez mais cedo. Como conseqüência, observou-se um crescente aumento nos programas de intervenção precoce e, também, no desenvolvimen-

\footnotetext{
* Licenciada em Psicologia/UNESP/Bauru e bolsista de Iniciação Científica FAPESP. Endereço: Rua Henrique Savi, 14-44 Apto 601, Jardim Infante Dom Henrique Bauru, SP, CEP: 17 012-205 Fone: (14) 32345753 E-mail: dorataques@yahoo.com.br

** Doutora em Psicologia Experimental, docente do Curso de Psicologia/UNESP/Bauru Endereço: Rua Pelegrino Constanzo, 2-62, Samambaia Residencial Park, Bauru, SP CEP: 17 018-110, Fone: (14) 3234 2237. E-mail: olgarolim@fc.unesp.br
} 
to de instrumentos de identificação das defasagens apresentadas por bebês. Esse crescimento de programas de intervenção voltados ao desenvolvimento infantil se justifica já que os cuidados despendidos aos bebês, no sentido de neutralizar riscos e evitar que estes se perpetuem, serão tão mais eficientes quanto mais precoce for a identificação das variáveis que afetam o desenvolvimento pleno da criança ${ }^{1}$. Como condições adversas, podemos considerar a influência de fatores ambientais, biológicos ou até a combinação destes dois, o que caracterizaria uma etiologia multifatorial, podendo estar presente antes, durante e após seu nascimento ${ }^{2}$. São os chamados fatores de risco.

O conceito de risco está colocado na medida em que os bebês, em determinadas condições biológicas e/ou ambientais, têm maior probabilidade de apresentar distúrbios ou atrasos em seu desenvolvimento quando comparados a bebês que não sofreram a influência das mesmas variáveis ${ }^{3}$. Linhares ${ }^{1}$ aponta que essas variáveis devem ser entendidas enquanto possibilidade, e não certeza, de que essa exposição trará prejuízos para o desenvolvimento do bebê, chamando-os de "fatores de risco em potencial”. Tais fatores ou agentes, se presentes, têm ação desconhecida sobre o desenvolvimento de um embrião, feto ou bebê. Os efeitos dependem da idade no momento da exposição, da quantidade do agente, da predisposição genética e do mecanismo patogênico específico de cada agente teratogênico. Estas va1riáveis têm, então, peso relativo dificultando a identificação segura do agente e dos efeitos da sua ação ${ }^{4}$.

Alguns estudos mais recentes têm contribuído para esclarecer questões referentes ao desenvolvimento de crianças nascidas prematuramente. McGrath (apud ${ }^{1}$ ) realizou um acompanhamento longitudinal de crianças nascidas prematuras, subdividas em grupos conforme as condições antes do nascimento e a evolução clínica, comparadas com crianças nascidas a termo. Verificou que a condição de prematuridade no nascimento relacionava-se significativamente com o desenvolvimento neurológico posterior da criança, mediando problemas de desenvolvimento cognitivo quando essas já se encontravam com oito anos de idade. Igualmente, um estudo de Hack (apud ${ }^{1}$ ) descreveu o acompanhamento até os 20 anos de idade, de bebês nascidos com uma condição baixo peso. O autor encontrou que esses bebês apresentavam maior probabilidade de ter um ou mais problemas de saúde crônico, como surdez, cegueira ou paralisia cerebral. Além disso, quando avaliados aos oito anos de idade, esses bebês apresentaram escores de QI mais baixos e desempenho acadêmico pior que bebês nascidos com peso normal. Estudos brasileiros também confirmam este quadro atual; como exemplo, o estudo feito por Vazques (apud ${ }^{5}$ ) em que trinta crianças nascidas pré-termo e com baixo peso foram comparadas com 28 crianças nascidas a termo com relação ao vocabulário expressivo, aos três anos de idade, utilizando a LAVE (um instrumento para avaliar a produção de palavras). Os resultados mostraram que o primeiro grupo produziu significativamente menos palavras do que o segundo grupo. Um outro levantamento feito em um serviço de Psicologia da Unifesp ${ }^{5}$ verificou que $29 \%$ de adolescentes atendidos com queixa de problema escolar tiveram problemas de gestação e parto como prematuridade, asfixia, infecção hospitalar, eclampsia.

A avaliação do desenvolvimento de bebês em situação de risco tem favorecido a implementação de programas de estimulação precoce, prevendo atrasos posteriores de desenvolvimento para as crianças com inabilidades estabelecidas, qual seja, aquelas que, ao nascer, já tem identificado uma condição limitadora, de acordo com definição de Guralnick ${ }^{6}$. Essas intervenções são implementadas no sentido de minimizar problemas que podem comprometer irremediavelmente seu desenvolvimento. 
A partir da constatação da possibilidade de risco, a criança deve ser encaminhada para a avaliação do desenvolvimento.

O diagnóstico precoce de fatores de risco, tanto biológicos quanto ambientais, é de suma importância para a identificação de crianças que necessitam de acompanhamento mais cuidadoso para alcançar o nível máximo de desenvolvimento de suas potencialidades cognitivas, afetivas, motoras e sociais. No caso de bebês de risco, há a probabilidade de que a condição de deficiência não exista, mas não é possível avaliar o seu alcance. A estimulação precoce deve atuar na prevenção ou tratamento de possíveis ocorrências futuras de distúrbios psiquiátricos graves, problemas médicos crônicos, atraso no desenvolvimento, transtornos de aprendizagem e outros problemas graves $^{7}$. Como tais ocorrências futuras de problemas no desenvolvimento dos bebês têm alto custo tanto para o próprio indivíduo, quanto para as áreas de Saúde e Educação, a implementação de medidas preventivas deve fornecer respostas adequadas de suporte ao desenvolvimento e à qualidade de vida dos bebês, indo além da simples questão de sobrevivência dos mesmos ${ }^{1}$.

As ações preventivas têm como objetivo oportunizar o desenvolvimento adequado de crianças. Um dos meios para atingir a estimulação adequada é o fornecimento de orientação aos pais, de modo a ensiná-los a estimular seu bebê em casa de acordo com sua realidade e as necessidades do filho, respeitando o ritmo de aprendizagem e a fase de desenvolvimento do mesmo. Para isso, é fundamental que as pessoas que estão em contato com o bebê conheçam as principais etapas de seu desenvolvimento para poderem estimular, de maneira efetiva, todo o potencial do qual é dotado ${ }^{8}$.

Um dos objetivos dos programas de estimulação precoce é intensificar as interações entre o bebê e os adultos significativos, identificando as áreas deficientes do desenvolvimento e promovendo um ambiente sadio e estimulador a partir do planejamento de intervenções que recuperem as deficiências observadas e atuem no sentido de estimular comportamentos que ocorram o mais próximo possível do esperado para a idade cronológica.

No Brasil, de acordo com pesquisas descritas por Martins e cols. ${ }^{9}$ e por Souza e cols. ${ }^{10}$, há uma alta incidência de nascimentos de bebês prematuros e de baixo peso. Os programas de intervenção junto aos pais de bebês prematuros e de baixo peso têm se mostrado eficazes em ensinar mães a observarem e interpretarem os comportamentos de seus filhos, tornandoos mais capazes de modificarem suas ações contingentemente às necessidades de desenvolvimento do bebê, favorecendo, assim, interações mais sincrônicas e recíprocas ${ }^{11}$.

Além dos bebês prematuros e de baixo peso, há uma incidência ainda maior de crianças e adolescentes, na faixa etária de 10 a 18 anos, tornando-se mães. A gravidez em mulheres muito jovens apresenta risco não só para os bebês, como para elas próprias. As conseqüências vão de danos biológicos, já que mãe e filho concorrem para a absorção de alimentos por estarem ambos em fase de desenvolvimento, até danos emocionais pela imaturidade da mãe ${ }^{12}$.

Inúmeras são as escalas para avaliação de desenvolvimento infantil disponíveis e a escolha deve se pautar: pelo objetivo da avaliação, pela idade da criança e pela disponibilidade de pessoal especializado em sua aplicação ou interesse dos envolvidos em desenvolver as habilidades necessárias e indispensáveis para a utilização do material de acordo com as normas padronizadas e/ou descritas nos manuais de aplicação. Um outro fator importante é a disponibilidade pessoal e psicológica dos cuidadores significativos se envolverem nos procedimentos de avaliação e intervenção, necessários para garantir a adequabilidade do desenvolvimento da criança ${ }^{13}$. 
Um método de avaliação de desenvolvimento utilizado em larga escala é a Escala de Desenvolvimento de Gesell. Este instrumento divide sua avaliação em quatro aspectos: comportamento motor, comportamento adaptativo, comportamento de linguagem e comportamento pessoal-social. Estes aspectos são analisados em idades cronológicas específicas: 4 semanas, 16 semanas, 28 semanas, 40 semanas, 12 meses, 18 meses, 24 meses e 36 meses. $\mathrm{O}$ resultado final é quantitativo e expresso como quociente de desenvolvimento (QD). Quando a criança é prematura, deve ser utilizada a idade corrigida, principalmente no primeiro ano de vida. Neste caso, o acompanhamento não é feito pontualmente, mês a mês, e também não se considera o sexo da criança ${ }^{14}$.

Há, ainda, instrumentos voltados para triagem de bebês com anormalidades em seu desenvolvimento, como o Denver, utilizado em crianças de 15 dias a 6 anos de idade, consideradas ou não de risco. Aqui também são consideradas quatro áreas: motora grosseira, motora fina-adaptativa, pessoal-social e linguagem. A vantagem deste teste é sua praticidade na aplicação. Entretanto, ocorre na utilização desta escala uma discrepância quando aplicada em prematuros, pois ao se considerar a idade cronológica, o desempenho parece ser muito prejudicado pela questão do risco e quando se considera a idade corrigida, o desempenho fica muito acima, acabando por desprezar a condição de risco ${ }^{15}$. Magalhães e cols. ${ }^{16}$ usaram o Teste de Desenvolvimento de Denver para avaliar o desempenho de crianças nascidas prematuras, nas áreas de linguagem, social-pessoal, motor fino adaptativo e motor amplo, nas idades de 12, 18 e 24 meses. Os resultados obtidos mostraram que fatores culturais podem ter contribuído para o baixo desempenho em todas as áreas dos sujeitos avaliados, recomendando cautela no uso deste instrumento com crianças brasileiras. Carvalho e cols. ${ }^{17}$ (2002) utilizaram a Escala Denver II para avaliar o de- sempenho de crianças de zero a seis anos, filhos de mães de baixa renda que trabalham fora , e cujos filhos não freqüentam creche. Os resultados indicaram que as defasagens mais importantes foram encontradas nas áreas pessoal-social e da linguagem.

Outra escala encontrada como referência em muitos trabalhos de pesquisas é a Escala Bayley de Desenvolvimento Infantil. Inicialmente, quando criada, havia um destaque para a avaliação das habilidades motoras de crianças até a idade de 3 anos, sendo ampliada posteriormente. Atualmente, avalia crianças recémnascidas até 30 meses de idade. Considera três áreas: mental, psicomotora e comportamental. Este instrumento não é padronizado para a população brasileira mas, quando utilizada, tem sido útil no acompanhamento do desenvolvimento de crianças por facilitar orientações e intervenções junto aos cuidadores. No entanto, a escala considera que se pode determinar as prováveis interferências recebidas pela criança em seu desenvolvimento, especialmente as socioafetivas $^{18}$. Um estudo realizado por Goto e cols. ${ }^{19}$ procurou avaliar e comparar o neurodesenvolvimento de bebês nascidos a termo, com peso adequado (AIG) ou pequeno para a idade gestacional (PIG), no $2^{\circ}$ mês de vida. Avaliaram-se 67 lactentes: 43 AIG e 24 PIG, utilizando as Bayley Scales of Infant Development. Como resultado geral da pesquisa, pode-se ver que o Index Score (IS) nas Escalas Mental e Motora foi significativamente menor no grupo PIG. Entretanto, os autores referiram a pouca confiabilidade das Escalas Bayley, bem como de outros instrumentos similares, em predizer nessa faixa etária, o potencial desenvolvimento posterior.

Baseada em obras de Piaget, há a escala de desenvolvimento psicológico de bebês de Uzgiris e Hunt adaptada por Dunst. Este instrumento é composto por um conjunto de escalas criada para avaliar bebês de recém-nascidos até 2 anos de idade. Essas escalas determinam pon- 
tos fracos e fortes em sete áreas de domínio, considerando oito medidas. È uma escala bem diferente das escalas tradicionais, apresenta muita flexibilidade nos procedimentos e materiais necessários para sua aplicação. Porém, sua aplicação não apresenta muita praticidade ${ }^{20}$.

Recentemente uma escala, elaborada e padronizada no Brasil, propõe-se a avaliar, mês a mês, o primeiro ano de vida da criança, apontando comportamentos em defasagem, propiciando planejamento de procedimentos de intervenção: O Desenvolvimento da Criança no Primeiro Ano de Vida ${ }^{21}$. Este instrumento compreende 64 comportamentos que são avaliados mês a mês, utilizando protocolo de acordo com o sexo da criança, avaliada de acordo com a condição padronizada. Os comportamentos avaliados para cada idade podem estar dentro de alguns critérios: comportamentos que são considerados como aparecimento, quando o comportamento apareceu pelo menos para uma das crianças da amostra; normalização, quando o comportamento ocorreu para a média das crianças da amostra e estabilização, quando o comportamento ocorreu para a maioria das crianças da amostra. Um estudo feito com 44 sujeitos $^{22}$ (Francisco e col., 2002), considerando o seu desempenho no primeiro mês de vida, indicou que os meninos apresentavam desempenho abaixo do esperado, apontando para a necessidade de atenção e estimulação sistemática a esta população. Rodrigues e cols. ${ }^{23}$, analisando os efeitos da avaliação e da orientação dadas aos cuidadores, constataram que os pais relatam que passaram a observar mais os comportamentos dos seus bebês, sentindo-se mais competentes e informados a respeito de como ajudá-los a se desenvolverem de formas mais positivas. Todavia, a escala utilizada divide os comportamentos observados somente em duas categorias: comportamentos motores e psicológicos. A divisão dos comportamentos observados em áreas como, por exemplo, social, de linguagem, motora, etc., facilitaria a identifica- ção de repertórios a serem observados e a conseqüente escolha de estratégias para estimulálos ou encaminhá-los para atendimento especializado, se necessário.

Já o instrumento chileno "Escala de Avaliação do Desenvolvimento Psicomotor de 0 a 24 Meses” (EEDP), de Rodriguez e cols. ${ }^{24}$, distingue quatro áreas de funcionamento: motora, linguagem, social e coordenação. AEEDP mede o rendimento da criança frente a situações determinadas em que ela necessita de graduados níveis de desenvolvimento psicomotor para obter sucesso na realização das atividades avaliadas. Considera-se quinze grupos de idade entre 0 e 24 meses para a aplicação da escala: 1 , 2, 3, 4, 5, 6, 7, 8, 9, 10, 12, 15, 18, 21 e 24 meses. Estas foram as idades consideradas, pelo instrumento, as mais significativas na aquisição de novas condutas no decorrer do desenvolvimento de uma criança. A escala definitiva tem um total de 75 itens, sendo dividida em cinco itens para cada um dos grupos de idade, não prevendo a divisão por sexo. A avaliação do desempenho das crianças é realizada em termos de normalidade, risco ou com atraso no desenvolvimento. Melchiori e cols. ${ }^{25}$ utilizaram a EEDP para avaliar 98 bebês de creches e observaram que havia diferenças entre o desempenho de meninos e meninas e sugerem a utilização de medidas diferentes considerando o sexo do bebê.

O Inventário Portage Operacionalizado $(\text { IPO })^{26}$, recentemente adaptado para a realidade brasileira, é composto por 580 comportamentos distribuídos em cinco áreas, separados por faixa etária de zero a seis anos e uma área específica para bebês de zero a quatro meses. Não há avaliação de comportamentos específicos em cada idade considerando o sexo da criança. Rodrigues e col. ${ }^{27}$, utilizando o protocolo do Instrumento Portage Operacionalizado (IPO), analisaram os desempenhos de 16 bebês no primeiro mês de vida, levantando os comportamentos típicos de cada sexo e de cada 
mês. Os resultados mostraram que, da aplicação dos 45 comportamentos sugeridos pelo protocolo de Estimulação Infantil, 80\% ou mais das meninas apresentaram 16 dos comportamentos avaliados e $80 \%$ ou mais dos meninos apresentaram 20. Destes, oito comportamentos estavam presentes apenas no repertório das meninas e seis apenas no dos meninos. Os dados obtidos neste primeiro mês mostram que o desempenho dos meninos é diferente do das meninas, tanto na quantidade de comportamentos apresentados como na especificidade deles. A maioria dos meninos apresentou um número maior de comportamentos quando comparados com as meninas. Estes dados sugerem que o Instrumento Portage Operacionalizado, em especial o protocolo de Estimulação Infantil, deveria ser padronizado visando estabelecer, mês a mês, quais os comportamentos mais prováveis de ocorrer para cada sexo, de forma a conduzir uma avaliação mais objetiva, facilitando a elaboração de procedimentos de treino, que são dificultados quando o número de comportamentos avaliados é muito grande.

O presente projeto pretendeu ampliar os estudos feitos, tanto no número de sujeitos quanto no número de sessões realizadas, a partir da avaliação mensal do desenvolvimento de bebês, dos comportamentos previstos no protocolo de “Estimulação Infantil”, do IPO, identificando comportamentos típicos em cada mês até o quarto mês de vida.

\section{MÉTODO}

Participaram do projeto 40 crianças, sendo 22 meninos e 18 meninas que foram mensalmente avaliados numa sala para atendimento infantil em um Centro de Psicologia Aplicada, de uma Universidade pública do interior do Estado de São Paulo. Os bebês foram identificados em uma Maternidade local e seus pais foram convidados a participar do projeto. Por ocasião do convite, foi agendado o primeiro encontro no mês seguinte, em dia próximo à data de aniversário de um mês, para a primeira avaliação. Em caso de concordância, os pais assinavam o Termo de Consentimento Livre e Esclarecido. Era aplicado, inicialmente, o roteiro de anamnese e conduzida a primeira avaliação do desempenho da criança, de acordo com as instruções do manual de aplicação do IPO ${ }^{26}$, previsto para os quatro primeiro meses. Ainda neste encontro, era agendada a próxima avaliação por ocasião do aniversário de $2^{\circ}$ mês do bebêe, assim, foi feito o mesmo procedimento para as avaliações subseqüentes. As avaliações foram conduzidas na presença dos pais que serviram como mediadores e informantes.

O comportamento do bebê foi observado: a) a partir de estimulação ambiental (por exemplo: "comportamento de olhar em direção ao local de onde provém um ruído ou movimentar o corpo em resposta a sons", quando o pesquisador aciona chocalhos, bate palmas a certa distância do bebê); b) observação da emissão de comportamentos espontâneos: segurar a criança em posição vertical no colo e observar se ela levanta e mantém erguida momentaneamente a cabeça ou se ela movimenta os braços e pernas quando deitada; c) observações relatadas pelos pais: comportamentos na alimentação, sobre a rotina do sono, choro, se reconhecem os membros da família.

\section{RESULTADOS E DISCUSSÃO}

O presente projeto teve como objetivo verificar, a partir da avaliação mensal do desenvolvimento de bebês, se existem comportamentos típicos em cada mês. As análises realizadas baseiam-se nos conceitos de média e de maioria dos sujeitos partindo da distribuição normal, que pretende descrever, com exatidão, a freqüência de ocorrência de comportamentos para populações humanas. Com base na pro- 
posta de Garrett ${ }^{28}$, estabeleceu-se como aparecimento do comportamento quando menos de 24\% das crianças o apresentaram em cada mês; como normalização, quando $25 \%$ a $75 \%$ das crianças o apresentaram; e como estabilização, quando mais de 76\% das crianças apresentaram o comportamento avaliado.

O primeiro conjunto de dados pretendeu descrever e analisar, a cada mês, os 45 comportamentos avaliados dentro de cada uma das características descritas: aparecimento, normalização e estabilização de todos os bebês.

A Figura 1 mostra a freqüência dos 45 comportamentos do protocolo "Estimulação Infantil” coletados na primeira avaliação. Podemos observar que $25 \%$ dos comportamentos estavam presentes para todos os bebês, na condição de estabilizados. Deles, 42\% estavam presentes para a média dos bebês (entre 25 e 75\% dos bebês avaliados), 29\% estavam presentes para menos de $25 \%$ dos bebês e $4 \%$ dos comportamentos, nenhum bebê apresentou.

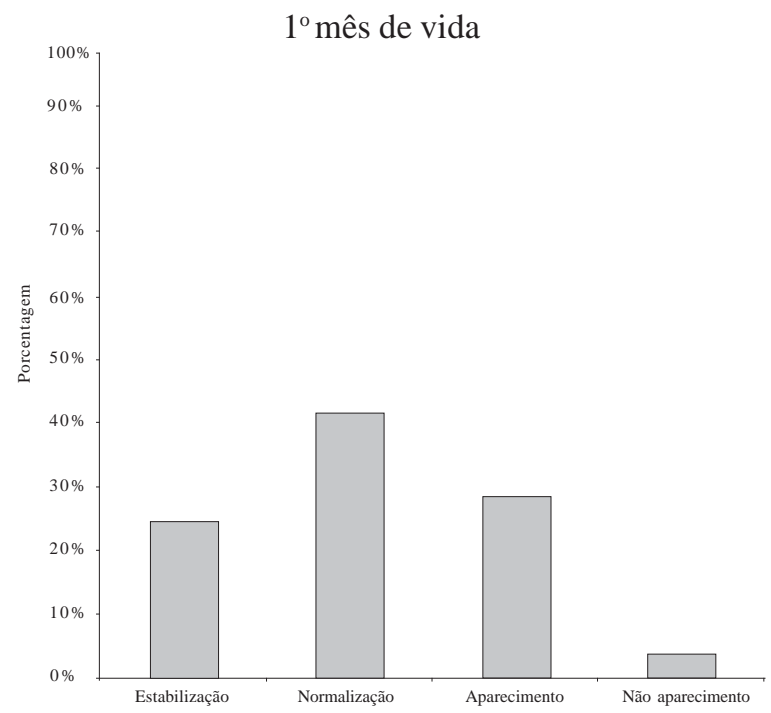

Figura 1: Freqüência dos comportamentos na primeira avaliação conduzida.

A Figura 2 mostra a freqüência dos 34 comportamentos do protocolo "Estimulação Infantil” avaliados na segunda avaliação, retirados os 11 comportamentos que já haviam sido estabilizados no primeiro mês. Podemos observar que, destes, $26 \%$ dos comportamentos estavam presentes para todos os bebês, na condição de estabilizados. 48\% estavam presentes para a média dos bebês (entre 25 e $75 \%$ dos bebês avaliados) e 26\% estavam presentes para menos de $25 \%$ dos bebês.

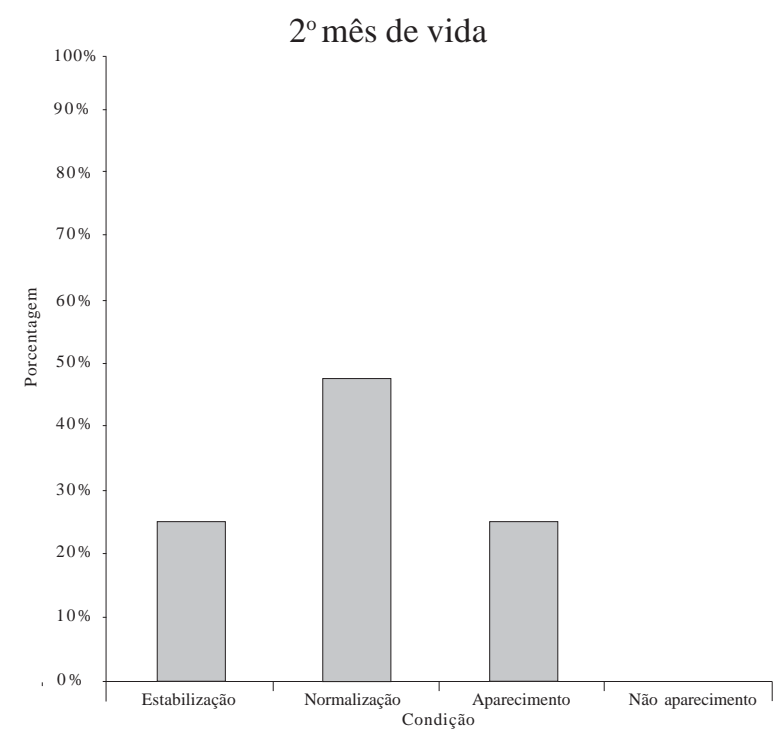

Figura 2: Freqüência dos comportamentos na segunda avaliação conduzida.

A Figura 3 mostra a freqüência dos 25 comportamentos do protocolo "Estimulação Infantil” avaliados na terceira avaliação, retirados os 20 comportamentos que já haviam sido estabilizados no primeiro e no segundo mês. Podemos observar que, destes, 36\% dos comportamentos estavam presentes para todos os bebês, na condição de estabilizados. 56\% estavam presentes para a média dos bebês (entre 25 e $75 \%$ dos bebês avaliados) e $8 \%$ estavam presentes para menos de $25 \%$ dos bebês.

A Figura 4 mostra a freqüência dos 16 comportamentos do protocolo "Estimulação Infantil” avaliados na quarta avaliação, retirados os 29 comportamentos que já haviam sido estabilizados no primeiro, segundo e no terceiro mês. Podemos observar que, destes, 44\% dos comportamentos estavam presentes para todos os bebês, na condição de estabilizados e 
$56 \%$ estavam presentes para a média dos bebês (entre 25 e $75 \%$ dos bebês avaliados).

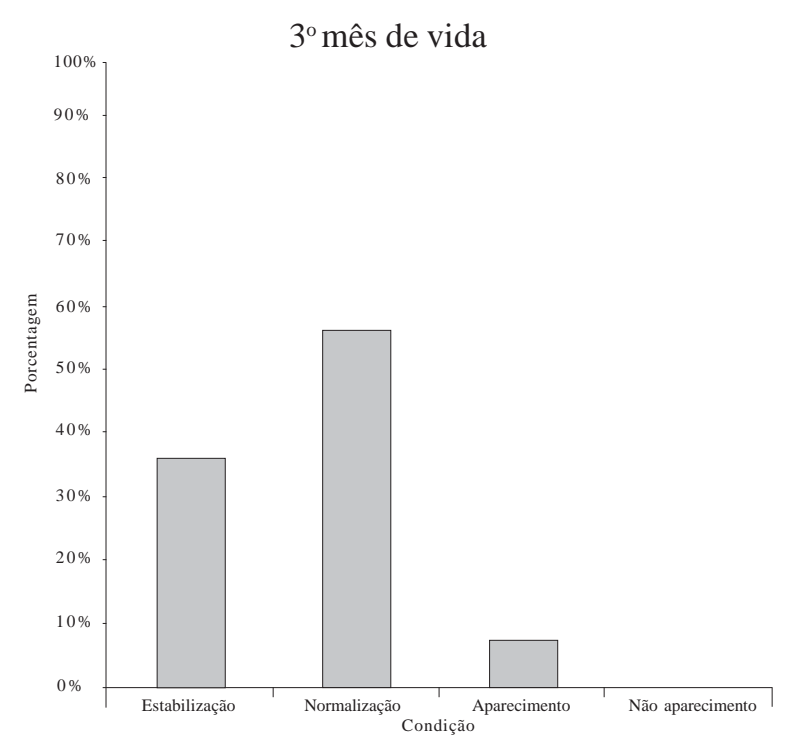

Figura 3: Freqüência dos comportamentos na terceira avaliação conduzida.

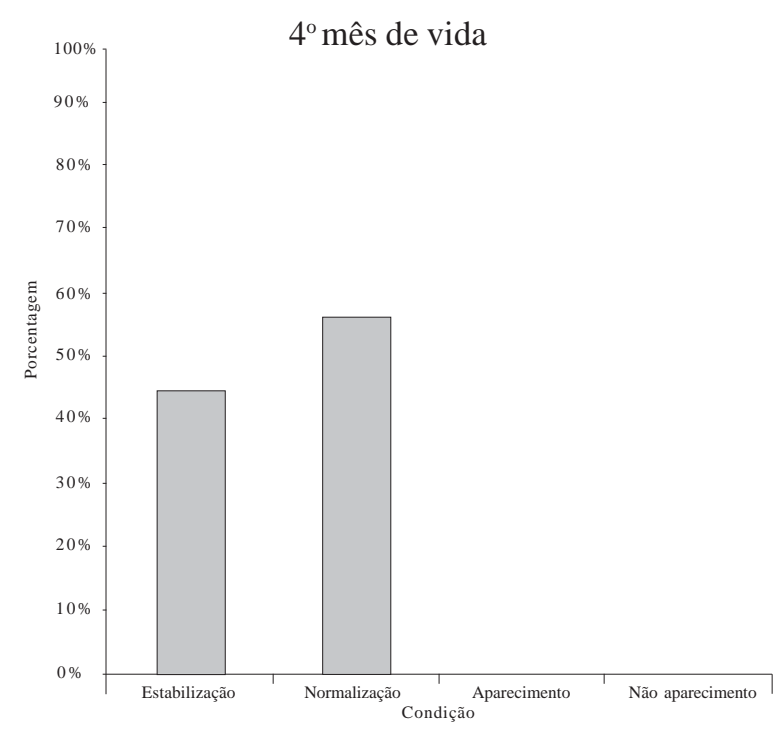

Figura 4: Freqüência dos comportamentos na quarta avaliação conduzida

O Inventário Portage Operacionalizado (IPO) ${ }^{26}$ prevê que todos os 45 comportamentos que compõem o protocolo de "Estimulação Infantil” possam ser avaliados já a partir do primeiro mês de vida da criança, uma vez que considera como critério de encerramento da aplicação que ela apresente 15 er- ros consecutivos nestes comportamentos a cada mês. Os dados obtidos mostraram que, mesmo no primeiro mês, a maioria dos bebês não apresentou 15 erros consecutivos, fazendo com que todo o protocolo tivesse que ser aplicado, segundo o critério proposto pelo instrumento. Este fato acaba resultando no inicio dos protocolos para cada área de zero a um ano, para alguns bebês, com o objetivo de cumprir o critério pré-estabelecido pelo instrumento.

Os dados apresentados nas Figuras $1 \mathrm{a}$ 4 parecem indicar que existem comportamentos típicos em cada mês. No primeiro mês, por exemplo, há uma porcentagem de 4\% de comportamentos ausentes para todos os bebês e há ainda uma porcentagem de 29\% de comportamentos que aparecem para poucos bebês. Diferenças no aparecimento, normalização e estabilização acabam aparecendo também nos meses subseqüentes como mostram as Figuras 2, 3 e 4. Sendo assim, o critério proposto inicialmente pelo instrumento de aplicação de todos os comportamentos parece não ser o mais adequado. Uma alternativa possível seria a aplicação, a cada mês, daqueles comportamentos que aparecem enquanto estabilizados, já que a maioria dos bebês deste grupo os apresentou, sugerindo que sejam necessariamente avaliados, assim como os comportamentos que a média dos bebês apresentou (normalizados) que também deveriam ser avaliados uma vez que têm alta probabilidade de ocorrência. Para estes últimos comportamentos, haveria uma informação adicional aos acompanhantes de que estes poderiam aparecer, mas caso não ocorram, não há motivo para preocupação, porém devem ser estimulados uma vez que deverão fazer parte do repertório do bebê já no próximo mês. Os comportamentos que estão na condição de aparecimento não precisariam ser avaliados já que foram apresentados por poucos bebês ou sua ocorrência foi relatada pelas mães pela impossibilidade de observação deste comportamen- 
to na situação de avaliação. Assim, o tempo gasto com a aplicação seria menor e as avaliações seriam mais pontuais.

Sendo a avaliação feita sempre na presença dos pais funcionando como informantes, além de diminuir o tempo gasto, a avaliação de repertórios com maiores chances de estarem presentes no repertório de bebês poderia diminuir a angústia nos pais, principalmente nos pais de primeiro filho. Uma avaliação mais sistemática também poderia contribuir para os projetos de orientação aos pais com relação ao desenvolvimento de suas crianças, já que é cada vez mais enfatizada na literatura especializada a relevância do envolvimento das famílias em programas de intervenção precoce ${ }^{7}$. Sabe-se que a participação efetiva dos pais deve ser garantida, considerando a importância do seu papel na implementação de práticas que podem otimizar o desenvolvimento de seus filhos. Os programas de estimulação precoce junto aos pais têm mostrado que as orientações feitas têm contribuído para interações mais sincrônicas e recíprocas entre cuidadores e bebês, possibilitando um desenvolvimento mais adequado dessas crianças. Dessa forma, a aplicação apenas dos comportamentos com maior possibilidade de ocorrer (estabilizados e normalizados) também facilitaria as orientações aos pais, já que se um comportamento que é estabilizado para a amostra não aparecesse no repertório de um bebê, o atraso poderia significar maior gravidade do que algum outro surge como normalizado para a amostra. Além disso, se os comportamentos que estão, nesta análise, identificados enquanto aparecimento não estivessem presentes no repertório de um bebê, a orientação com relação a qual área deveria ser estimulada viria acompanhada de uma explicação da orientadora no sentido de ressaltar que a estimulação daquela área não faria, necessariamente, com que ela se desenvolvesse logo, os pais devendo saber que poderia demorar mais tempo. Neste caso, as orientações poderiam se tornar mais sistemáti- cas, além de tornar os diagnósticos mais efetivos, fazendo com que os possíveis encaminhamentos fossem realizados o quanto antes, tornando mais eficientes os cuidados despendidos aos bebês, no sentido de neutralizar riscos e evitar que estes se perpetuem.

\section{CONSIDERAÇÕES FINAIS}

O Inventário Portage Operacionalizado (IPO) ${ }^{26}$ prevê a avaliação dos 45 comportamentos do protocolo de Estimulação Infantil, cumprindo o critério de sua suspensão pela inexistência de 15 comportamentos consecutivos no repertório da criança, não prevendo a avaliação de comportamentos específicos em cada idade. Autilização constante desta avaliação em programas de orientações de pais de bebês de risco mostrava que a avaliação de comportamentos com pouca chance de fazerem parte do repertório da criança nas idades iniciais resultava em ansiedade e angústia dos cuidadores. Esses verbalizavam a sua preocupação com um possível atraso no desenvolvimento de seu bebê, devido à possibilidade da ocorrência desse atraso pela condição de risco. Isto gerava, também, dificuldade para os aplicadores que tinham que argumentar sobre a necessidade de cumprir o critério do instrumento, fato além da realidade cotidiana dos pais. O presente projeto pretendeu, então, conduzir uma coleta de dados que permitisse identificar no Protocolo de “Estimulação Infantil” se havia comportamentos típicos a cada mês. Estabeleceram-se as condições de estabilização, normalização e aparecimento dos comportamentos a partir da sua freqüência a cada mês. Os resultados obtidos mostraram que há comportamentos típicos (estabilizados) e prováveis (normalizados) para cada mês. A utilização destes critérios deve resultar numa economia de tempo e desgaste emocional dos cuidadores e aplicadores. Um número menor de comportamentos avaliados 
facilitaria sobremaneira o processo de orientação de pais. Porém, tais resultados se referem a esta amostra estudada. Estudos conduzidos com populações maiores poderão validar estes resultados. Uma outra sugestão se refere à incorporação deste protocolo aos protocolos por área de zero a 1 ano, o que também, facilitaria a aplicação deste instrumento em serviços de orientação aos pais. O Inventário Portage Operacionalizado tem se mostrado um instrumento importante de avaliação de desenvolvimento de bebês, pela ampla gama de comportamentos avaliados, não presentes em outras escalas. Estudos que colaborem para torná-lo de mais fácil aplicação aumentariam a sua utilização em outros programas de estimulação precoce.

\begin{abstract}
The present project intended to evaluate the development of 40 babies, using Inventário Portage Operacionalizado (IPO), during the first four months of life. In this period the project evaluated the occurrence, in each month, of 45 behaviors listed in the protocol of Infant Stimulation. This evaluation intended to identify frequency differences of these behaviors in each month. The monthly evaluations occurred in a date close to each baby's month anniversary. The babies came from a maternity hospital that attends on users of the SUS (Brazil's National Health System). The obtained results pointed to the existence of typical (stabilized) behaviors and probable (normalized) behaviors for each month. For this analysis the criteria were: conditions of stabilization (if the behavior occurred in more than $75 \%$ of the sample), normalization (if $25 \%$ to $75 \%$ of the sample presented it) and appearance (less than $25 \%$ of the babies presented it) in each month. In the first month, the babies presented 25\% (stabilization) of the 45 evaluated behaviors. Of the remaining ones, the babies presented $26 \%$ in the second month. In the third month, of the behaviors that were still not stabilized the babies presented $36 \%$ and, in the fourth month, they presented $44 \%$ of the remaining ones. Such results allow us to conclude that a lower number of evaluated behaviors would facilitate the process of evaluation of babies and the orientation given to parents, resulting in economy of time and less stress for parents and caregivers. Studies conducted with bigger populations can validate these results.
\end{abstract}

Keywords: Inventário Portage Operacionalizado. Development of babies. Evaluation of development.

\section{REFERÊNCIAS}

1. Linhares MBM. Estresse, resiliência e cuidado no desenvolvimento de neonatos de alto risco. In: Temas em educação especial: avanços recentes. São Carlos: EDUFSCar; 2004. p. 315-24.

2. Sanseverino MTV, Spritzer DT, Schüler-Faccini L, organizadores. Manual de teratogênese. Porto Alegre: Universidade Federal do Rio Grande do Sul; 2001.

3. Nunes LRDP. Educação precoce para bebês de risco. In: Rangé B, organizador. Psicoterapia comportamental e cognitiva: pesquisa, prática, aplicações e problemas. Campinas: Editorial Psy II; 1998.

4. Schüler-Faccini L, Schvartzman L, Cecchin CR. Teratogênese humana e o SIAT. In: Sanseverino MTV, Spritzer DT, Schüler-Faccini L, organizadores. Manual de teratogênese. Porto Alegre: Universidade Federal do Rio
Grande do Sul; 2001.

5. Pedromônico MRM. Instrumentos de triagem e a vigilância do desenvolvimento da criança de 0 a 6 anos. In: Temas em educação especial: avanços recentes. São Carlos: EDUFSCar; 2004. p. 325-30.

6. Guralnick MJ, editor. The effetiveness of early intervention. Baltimore: P. H. Brookes Publishing; 1997.

7. Assumpção TM. Influência das condições ambientais no desenvolvimento precoce infantil. Infanto Rev Neuropsiquiatr Infânc Adolesc. 2002;10(2):72-8.

8. Franco M, Ardore M. Estimulação precoce. Integração (Brasília). 1996;7(16):15-8.

9. Martins DC, Mello DF, Scochi CGS. Crianças prematuras e de baixo peso ao nascer em famílias de baixo nível socioeconômico: uma revisão de literatura. Pediatr Mod. 2001:37(9) 2001:37(9):452-9.

10. Souza KZ, Gomes IMM, Cambraia DS, Semião 
FP, Alves MP, Brasileiro ON. A incidência de recém nascidos de risco no serviço de saúde pública de Pouso Alegre - MG. Pediatr Mod. 2001;37(3):61-74.

11. Alves PP, Ferreira MFR, Nunes LRDP, Oliveira MCB, Kaoru J, Epelboim S. O desenvolvimento cognitivo de bebês prematuros e alguns aspectos neuromotores associados. Pediatr Mod. 1997;33(7):511-34.

12. Negri EMP, Tanaka EDO. Orientação de mães adolescentes como mediadoras do processo de desenvolvimento de seus bebês de risco. In: Marquezine MC, Almeida MA, Tanaka EDO, organizadores. Perspectivas multidisciplinares em educação especial. Londrina: UEL; 2001.

13. Rodrigues OMPR. Bebês de risco e sua família: o trabalho preventivo. Temas Psicol. 2003;11(2):37-55.

14. Carelli C. Métodos de avaliação do desenvolvimento. [ S.I.]: Sociedade Brasileira de Pediatria. Disponível em:

http://www.sbp.com.br/ show_item2.cfm?id_categoria=24\&id_detalhe= 322\&tipo_detalhe=s. Acesso em 10 jul 2005.

15. Hassano AYS. Métodos de avaliação do desenvolvimento. [ S.I.]: Sociedade Brasileira de Pediatria. Disponível em: http://www.sbp.com.brshow_item2.cfm?id_ categoria=24\&id_detalhe=322\&tipo_detalhe=s. Acesso em: 10 jul. 2005, 13:27:40.

16. Magalhães LC, Barbosa VM, Araújo AR, Paixão ML, Figueiredo EM, Gontijo APB. Análise do desempenho de crianças pré-termo no teste de desenvolvimento de Denver nas idades de 12, 18 e 24 meses. Pediatria. 1999;21(4):330-9.

17. Carvalho AM, Machado MGM, Suyama E. Afetividade, cultura e cuidado: possibilidades para o desenvolvimento da criança. In: Leite SAS, organizador. Cultura, cognição e afetividade: a sociedade em movimento. São Paulo: Casa do Psicólogo; 2002. p. 38-67.

18. Morsch D. Métodos de avaliação do desenvolvimento. [ S.I.]: Sociedade Brasileira de Pediatria. Disponível em:

http://www.sbp.com.br/ show_item2.cfm?id_categoria=24\&id_detalhe= 322\&tipo_detalhe=s. Acesso em: 10 jul. 2005, 13:27:40.

19. Goto MMF, Gonçalves VMG, Netto AA, Morcillo AM, Moura-Ribeiro MVL.

Neurodesenvolvimento de lactentes nascidos a termo pequenos para a idade gestacional no segundo mês de vida. Arq Neuropsiquiatr [periódico na internet]. 2005[acesso em 11 jul 2005];63(1):75-82. Disponível em: http://www.scielo.br/pdf/anp/v63n1/23602.pdf.

20. Oliveira MCB, Nunes LROP. Métodos de avaliação do desenvolvimento. [ S.I.]: Sociedade Brasileira de Pediatria. Disponível em: http://www.sbp.com.br/ show_item2.cfm?id_categoria=24\&id_detalhe= 322\&tipo_detalhe=s. Acesso em: 10 jul. 2005, 13:27:40.

21. Pinto EB, Vilanova LCP, Vieira RM. O desenvolvimento da criança no primeiro ano de vida. São Paulo: Casa do Psicólogo; 1997.

22. Francisco LC, Farias MO, Freitas RMC, Pacini BA, Rodrigues OMPR. O desempenho de bebês de risco no primeiro mês de vida. In: Anais da $6^{a}$ Jornada de Educação Especial; 2002 Jun 3-6. Marília, Brasil. Marília: Fundepe; 2002. p. 100.

23. Rodrigues OMPR, Melchiori LE, Lemos AAP, Ferreira DGC, Paccini BA, Freitas RC, et al. Acompanhamento do desenvolvimento de bebês de risco: avaliação e orientação aos cuidadores. In: Anais do $2^{\circ}$ Congresso de Extensão Universitária da Unesp; 2002 nov 710. Bauru, Brasil. Bauru: Unesp; 2002. p. 103.

24. Rodriguez S, Arancibia V, Undurraga C. Escala de evaluaciom del desarrollo psicomotor de 0-24 meses. $7^{\mathrm{a}}$ ed. Santiago: Editorial Galdoc; 1992.

25. Melchiori LE, Rodrigues OMPR, Alves CO, Andrieto E, Amaral JN. Há diferenças de gênero em relação ao desenvolvimento de bebês que freqüentam creches? In: Resumos da $33^{\text {a }}$ Reunião Anual de Psicologia da Sociedade Brasileira de Psicologia; 2003 out 22-26; Belo Horizonte, Brasil. Belo Horizonte: SBP; 2003. p. 163.

26. Willians LA, Aiello ALR. Inventário Portage operacionalizado. São Paulo: Mennon; 2001.

27. Rodrigues OMPR, Lemos AAP, Taques DCSR, Gigliotti CM, Mastine IL. Avaliação do repertório comportamental de bebês no primeiro mês de vida: uma proposta de análise. In: Caderno de resumos da $5^{\text {a }}$ Jornada de Psicossomática e Psicologia Hospitalar e I Encontro de Psicologia da Saúde; 2003 set 1920; Bauru, Brasil. Bauru: Unesp; 2003. p. 35.

28. Garret HE. Estatística en psicología y education. Buenos Aires: Paidós; 1974.

Recebido em 00/00/2006 Modificado em 05/07/2006 Aprovado em 15/07/2006 